\title{
Traces of the formation history of the Milky Way
}

\author{
B. Nordström ${ }^{1}$, E. Stonkute் $\dot{\mathrm{S}}^{2}$, \\ R. Ženovienè ${ }^{2}$ and G. Tautvaišienè ${ }^{2}$ \\ ${ }^{1}$ Niels Bohr Institute, University of Copenhagen, \\ Juliane Maries vej 30, DK-2100, Copenhagen, Denmark \\ email: birgitta@nbi.ku.dk \\ ${ }^{2}$ Institute of Theoretical Physics and Astronomy, \\ Vilnius University, Lithuania \\ email: edita.stonkute, renata.zenoviene, grazina.tautvaisiene@tfai.vu.lt
}

\begin{abstract}
Chemical and kinematical information is needed in order to understand and trace the formation history of our Galaxy. In the homogeneous large sample of $\mathrm{F}$ and $\mathrm{G}$ stars in the survey by Nordström et al. (2004), groups of stars with orbital parameters different from field stars were found by Helmi et al. (2006). Simulations of disrupted satellites showed that the groups had similar properties as infalling dwarf satellites would have after several Gyr. From high resolution spectra, we analyse elemental abundances of stars in 3 such groups with conserved kinematic properties. Here we present first results of s- and r- process element abundances in two such groups and compare with average field stars.
\end{abstract}

Keywords. Galaxy: formation, Galaxy: disk, stars: abundances, etc.

\section{Results and Conclusions}

Using high-resolution spectra, we measured elemental abundances of neutron-capture elements in stars attributed to two newly identified kinematic groups of the GenevaCopenhagen survey (GCS), which were suspected to be remnants of disrupted satellite galaxies. Our study of 32 stars in Group 2 and 20 stars in Group 3 shows the following:

I. The chemical composition of the stars within each group is homogeneous.

II. The abundances of chemical elements produced predominantly by the r-process are overabundant in comparison with Galactic thin-disk dwarfs of the same metallicity. The most prominent overabundances are seen for europium, samarium, and praseodymium.

III. The abundances of chemical elements produced mainly by the s-process are similar to those in the Galactic thin-disk dwarfs of the same metallicity.

IV. The chemical composition of stars is similar to thick-disk stars, which might suggest that their formation histories are linked.

The identification of such kinematic groups and the exploration of their chemical composition will be a key in understanding the formation and evolution of the Galaxy. One more kinematic group of the GCS survey will be analysed in a forthcoming paper.

Acknowledgements. The FIES spectrograph at the Nordic Optical Telescope was used to collect the data. BN is grateful to the Danish Research council for support.

\section{References}

Nordström, B., Mayor, M., Andersen, J., et al. 2004, A\&SA, 418, 989

Helmi, A., Navarro, J. F., Nordström, B., et al. 2006, MNRAS, 365, 1309 